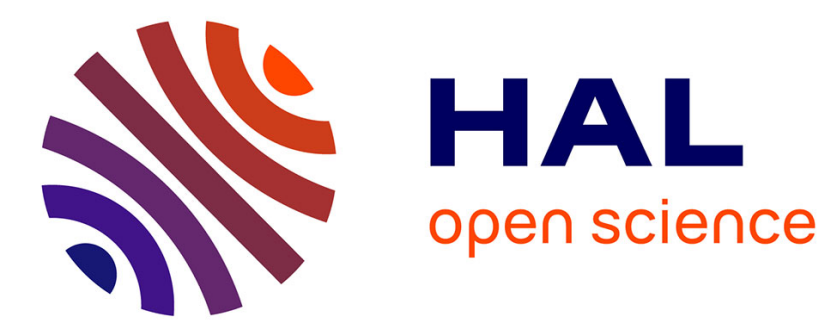

\title{
Participation journalistique sur Twitter. Injonctions à la participation et formes d'intervention numériques: le cas de la Radio Télévision Suisse
}

\author{
Bruno Asdourian, Florence van Hove, Dominique Bourgeois
}

\section{To cite this version:}

Bruno Asdourian, Florence van Hove, Dominique Bourgeois. Participation journalistique sur Twitter. Injonctions à la participation et formes d'intervention numériques : le cas de la Radio Télévision Suisse. Studies in Communication Sciences, 2015, 15, pp.190 - 196. hal-01859462

HAL Id: hal-01859462

https://hal.science/hal-01859462

Submitted on 22 Aug 2018

HAL is a multi-disciplinary open access archive for the deposit and dissemination of scientific research documents, whether they are published or not. The documents may come from teaching and research institutions in France or abroad, or from public or private research centers.
L'archive ouverte pluridisciplinaire HAL, est destinée au dépôt et à la diffusion de documents scientifiques de niveau recherche, publiés ou non, émanant des établissements d'enseignement et de recherche français ou étrangers, des laboratoires publics ou privés. 


\title{
Participation journalistique sur Twitter. Injonctions à la participation et formes d'intervention numériques : le cas de la Radio Télévision Suisse.
}

\author{
Bruno Asdourian, Florence Van Hove, Dominique Bourgeois \\ Université de Fribourg
}

Asdourian B., Van Hove F., Bourgeois D. (2015), Participation journalistique sur Twitter. Injonctions à la participation et formes d'intervention numériques : le cas de la Radio Télévision Suisse, Studies in Communication Sciences, 15(2), 190-196.

Résumé :

Cette recherche questionne la participation journalistique sur Twitter en observant les pratiques des acteurs médiatiques de la Radio Télévision Suisse (RTS) et celles des citoyens. Les critères d'analyse sont liés aux injonctions à la participation journalistique et aux formes d'intervention numériques. Une analyse de contenu de tweets $(\mathrm{N}=695)$ est menée sur deux lieux de discussion. L'analyse des messages publiés a révélé comment des acteurs médiatiques et des acteurs non médiatiques pouvaient cohabiter en ligne pour : (i) solliciter la participation journalistique ; (ii) participer à la création de contenus ; et (iii) participer aux interventions numériques en ligne.

Mots-clés : participation journalistique, Twitter, injonction, intervention, télévision. 


\section{Introduction}

Parmi les médias sociaux en ligne, les médias traditionnels et les journalistes utilisent majoritairement le réseau social Twitter (Revers, 2014). L'objectif est de diffuser et de relayer des informations mais aussi de recueillir des témoignages et des avis de citoyens. Pour ce faire, les médias télévisés tentent de guider les activités des récepteurs sur leurs propres médias sociaux en ligne à travers des injonctions à participer. Twitter est alors utilisé comme un outil technique numérique permettant aux médias de s'inscrire dans un modèle participatif ouvert aux formes de co-construction des informations relatives à l'actualité (Java, Song, Finin, \& Tseng 2007). Cette tendance vers une conception socio-technique (Rojot, 2005) des relations avec les citoyens favorise la rencontre entre les acteurs médiatiques et les internautes intéressés par une participation journalistique.

Les questions liées à ces usages de Twitter et aux modifications conceptuelles qu'ils favorisent occupent la recherche empirique récente sur le journalisme numérique (Hermida, 2013 ; Mercier \& Pignard-Cheynel, 2014). Néanmoins, la participation journalistique sur Twitter en Suisse romande constitue un terrain de recherche peu étudié. La présente recherche propose de pallier à ce manque en présentant une étude des activités ayant lieu sur des espaces de diffusion d'information et de discussion créées par la Radio Télévision Suisse (RTS) sur Twitter.

Les questions de recherche sont les suivantes :

- (QR1) Quels sont les différents acteurs participant à la diffusion de contenus sur ces espaces ?

- (QR2) Quels sont les contenus privilégiés par les acteurs médiatiques et par les citoyens ?

- (QR3) Quelles sont les formes de participation et d'interaction entre ces acteurs ?

Après une première section développant une revue de la littérature, nous présenterons notre étude de cas ainsi que la méthodologie de recherche. Nous discuterons ensuite des résultats principaux de notre étude et terminerons par une conclusion.

\section{Revue de la littérature}

\subsection{Les évolutions organisationnelles et relationnelles liées à l'appropriation des nouveaux médias par les médias traditionnels}

La littérature académique récente liée à la montée en puissance des usages des médias sociaux par les professionnels de la télévision a été essentiellement orientée selon deux axes : d'une part, l'étude des changements structuraux dans la production des contenus ; et d'autre part, l'étude de l'appropriation des médias sociaux par les praticiens de l'information télévisuelle. Le premier champ de recherche décrit, par exemple, la mise en place de newsrooms : des auteurs comme Zeller et Hermida (2015) ou Kredens et Rio (2015) évoquent, en effet, les nécessaires reconfigurations organisationnelles liées au traitement de l'information télévisuelle à l'ère du numérique. De nouvelles formes de discours télévisuels émergent ainsi afin de rassembler des audiences disséminées sur Internet, Twitter ou via les smartphones (Jeanne-Perrier, 2010, 2012 ; Sonnet, 2015). Le second champ de recherche, qui est centré sur les pratiques, met en avant les notions de lien (Jeanne-Perrier, 2015), de sociabilité (Jouët, 2015) et d'interaction (Pélissier \& Diallo, 2013) en analysant l'activité des parties prenantes sur les réseaux sociaux numériques (Badillo, 2015). Les «journalistes $2.0 »$ sont alors amenés à être plus fréquemment confrontés à la participation des citoyens à travers les avis, les informations et les commentaires laissés en ligne et, notamment, sur les outils de micro-blogging (Rebillard \& Touboul, 2010 ; Touboul, 2010). 


\subsection{Micro-blogging et activation de la participation journalistique}

Twitter est un outil de micro-publications caractérisé par l'émission en temps réel de courts messages de 140 caractères. Boyd, Golder et Lotan (2010) le décrivent comme un système d' " écologie conversationnelle ». En effet, Twitter fait réagir «des dizaines de millions de personnes dans le monde [...] sur des questions d'actualité politique, sociale, culturelle, économique, etc. » (Rieder \& Smyrnaios, 2012, p. 107). L'utilisateur de Twitter est ainsi présent parmi un réseau d'acteurs sociaux en ligne. Il est amené à rendre visible son point de vue à d'autres internautes et notamment aux médias traditionnels. Marres (2012) indique que Twitter permet ainsi d'activer des pratiques participatives.

Selon Galibert (2013), le concept de participation sous-entend l'engagement du citoyen dans l'action collective délibérative. Il existe de multiples formes de participation : l'inscription aux outils d'accès au débat ; la lecture passive ; ou l'intervention discursive. Ces formes de participation sont liées aux différents niveaux d'engagement souhaités par les internautes. L'objectif pour les médias traditionnels est alors de tenter d'attirer cette expression citoyenne créatrice de liens et/ou de contenus (Kozinets, 2010) sur leurs propres espaces numériques. Les usages de Twitter par ces médias traditionnels sont ainsi orientés à la fois vers la promotion, la couverture des événements, l'investigation, mais également vers les interactions et le monitoring de l'évolution de cette relation (Neuberger, Vom Hofe \& Nuernbergk, 2014). Il existe alors un renouvellement des rites relationnels avec les internautes.

Dans ce contexte, les auteurs de cette recherche proposent le concept de participation journalistique en référence aux travaux liés au concept de la participation politique (Monnoyer-Smith \& Wojcik, 2014), afin d'explorer les formes d'incitation à la participation et également les types de participation de citoyens qui s'inscrivent dans « une nouvelle culture participative sur le net »(Blondiaux \& Fourniau, 2011).

\subsection{Formes d'incitation à la participation}

L'injonction à la participation est composée de trois niveaux : information (acceptation du support) ; connaissance (lecture passive) ; et actions (intervention discursive) (Galibert, 2010). Ces formes d'incitation à participer ne sont pas nouvelles : «la part de production (de co-production) des « récepteurs/consommateurs » des médias traditionnels a toujours été présente, et ce dès les origines de la presse. [Ainsi,] l'histoire de la télévision a toujours connu des formes de participation des spectateurs » (Coutant \& Stenger, 2012, p. 79). Cependant, de nouveaux espaces d'incitation apparaissent en lien avec le développement des nouveaux médias et du web 2.0 : formulaires de proposition de sujets d'enquête sur les sites internet des médias traditionnels ; création de motsdièses Twitter pour l'envoi d'images d'un évènement en direct; boutons de lien vers les médias sociaux, etc. Dans le cas de la participation sur plusieurs écrans (Jeanne-Perrier, 2010), il existe également une forme active de participation individuelle à la conversation qui est organisée sur les médias sociaux à partir de la fourniture d'un sujet de conversation par la télévision. C'est en ce sens que la télévision s'inscrit dans une récente approche de télévision sociale (Proulx \& Shepatin, 2012).

D'une manière générale, même si l'injonction à la participation est issue d'un imaginaire d'Internet lié aux actions collectives délibératives (Galibert, 2013), elle reste, en fait, fortement liée à une action stratégique. Le contexte numérique contraint les médias traditionnels a de profondes modifications organisationnelles: les gestionnaires des médias traditionnels doivent se «faire aimer » par les internautes et doivent les «faire agir» sur les médias sociaux (Galibert, 2013). Cette ouverture des espaces d'expression des médias traditionnels au plus grand nombre vise à inclure l'avis de tous ceux qui souhaitent faire entendre leur voix. Ainsi, Galibert (2010) indique que les 
médias traditionnels répondent à la fois à leurs propres besoins d'animation de l'information et également aux besoins des usagers d'Internet de participer à « l'action collective ». Il est alors utile d'observer comment cette action liée à l'impératif délibératif (Blondiaux \& Sintomer, 2002) s'inscrit dans les outils sociaux mis en place par un média traditionnel.

\subsection{Formes de participation des journalistes et des citoyens}

Dans le contexte du journalisme en ligne, des études ont montré que les usages des médias sociaux par les journalistes étaient professionnels et «normalisés », c'est-à-dire majoritairement conformes aux normes et standards journalistiques établis (Singer, 2005 ; Hermida, 2010 ; Lasorsa, 2012). En ce qui concerne les usages de Twitter en particulier, il a été démontré que les journalistes se servent également de cet outil pour des usages plus personnels, dont le partage d'opinions et d'informations sur leurs activités journalistiques en général, signe d'une plus grande transparence envers les internautes (Lasorsa, Lewis \& Holton, 2012 ; Noguera-Vivo, 2013 ; Mercier \& Pignard-Cheynel, 2014 ; Revers, 2014). Hedman \& Djerf-Pierre (2013) indiquent quant à eux trois dimensions d'usage des réseaux sociaux par les journalistes : surveillance et information ; demandes organisationnelles ; réseautage et branding [notre traduction] (Hedman \& Djerf-Pierre, 2013 : p. 377). Dès lors, il semble que les journalistes adaptent désormais aussi leurs pratiques journalistiques à la logique des médias sociaux.

Les pratiques participatives des internautes sur les médias sociaux en ligne sont variées (Bourgeois, Clavien, Asdourian \& Van Hove, 2014) et ne se développent pas obligatoirement dans un objectif d'engagement long. Initialement, le rôle des médias sociaux a été observé sous l'approche de la révolution numérique (Monnoyer-Smith \& Wojcik, 2014), lorsqu'ils prédisent, notamment, de profonds bouleversements des pratiques journalistiques en lien avec la participation des internautes. Twitter est à la source de cette approche car il permet d'établir une communication interpersonnelle, de produire et diffuser du contenu en temps réel et à grande échelle (Bruns \& Burgess, 2012). Néanmoins, de récents travaux minorent la vision d'un bouleversement des pratiques journalistiques liées à la prise de pouvoir d'internautes désormais actifs. L'activité elle-même des internautes est discutée : la visibilité de leurs contenus rend, en effet, plutôt compte d'un désir individuel d'affichage de son identité digitale (Cardon, 2010 ; Lardellier \& Bryon-Portet, 2010) avec la création de contenus ayant les attributs d'un simple prétexte à la visibilité (Lahire, 2004).

Ainsi, les écrits sont plutôt de simples réactions sans lien avec le fil de discussion (Dupret, Klaus \& Ghazzal, 2010). Selon Boullier (2004), lorsque les internautes parlent entre eux des contenus des médias, les diverses « opinion[s] publique[s] locale[s] [...] permettent à l'opinion publique d'exister comme agrégat »(Boullier, 2004, p. 74). En outre, la visibilité des énoncés présents sur des médias sociaux comme Twitter participent, selon Badillo, Bourgeois et Asdourian « à une plus grande prise en compte des particularités locales » (Badillo, Bourgeois \& Asdourian, 2010, p. 179). Les opinions exprimées peuvent même fréquemment aller dans le sens d'une radicalisation et créer des groupes de pensée identique (Sunstein, 2001 ; Flichy, 2008 ; Coleman, 2012).

Au-delà de l'approche révolution/normalisation des médias sociaux en relation avec les pratiques journalistiques, il est désormais plus utile de considérer les manières dont le public s'autorise à intervenir, et ceci, plus particulièrement dans un contenu journalistique.

Nous allons, dès lors, examiner les supports matériels des publics participants, des contenus ainsi que des formes d'injonctions à agir dans un contexte de participation journalistique sollicitée par les médias traditionnels.

\section{Etude de cas et méthodologie}


Le cas d'étude est celui de la Radio Télévision Suisse (RTS). Bien qu'étant en concurrence avec les chaînes françaises, ce média public généraliste est le premier média audiovisuel de Suisse romande. En 2014, avec 2000 collaborateurs ${ }^{1}$, la RTS attire la plus large audience de la Suisse romande : $35 \%$ du marché de la télévision et $57 \%$ du marché de la radio ${ }^{2}$. La RTS est l'unité francophone de la Société Suisse de Radiodiffusion et Télévision (SRG SSR). Avec 17 stations radio, 7 chaînes TV ainsi que des sites Web et des services de télétexte, cette maison mère est la plus grande entreprise de médias électroniques de Suisse. La SSR compte également des unités dédiées aux trois autres régions linguistiques et à l'information internationale en ligne ${ }^{3}$. Dans le paysage médiatique suisse, la SSR est à la fois en concurrence intermédias « avec une cinquantaine de radios et TV privées locales ou régionales », et également intramédias «avec la multiplication des contenus dans une même famille médiatique » (Amez-Droz, 2015, p.93-99).

La RTS dispose d'une large offre d'outils numériques : en complément d'un site web, elle propose des applications pour supports mobiles et des espaces d'interaction sur les médias sociaux. La RTS anime ainsi une soixantaine de pages Facebook et près de trente comptes sur Twitter. Ainsi, les enjeux de la RTS face au contexte numérique, et plus particulièrement du point de vue de la gestion de l'information, sont liés à une lutte contre la fragmentation des audiences et une adaptation face à des usages de multiples écrans. Un des objectifs est alors d'élaborer des formats informationnels favorisant, d'une part, la consultation en rattrapage, et d'autre part, les interactions et la participation du public ${ }^{4}$.

Deux lieux de discussion proposés par la Radio Télévision Suisse sur Twitter ont été retenus : les messages contenant le hashtag \#19h30; et les messages liés au fil de discussion @RTSinfo. Selon les chiffres de la RTS, en 2014, le téléjournal «Le 19h30 » représente 60\% de part de marché en Suisse romande ${ }^{5}$ et le compte Twitter @ RTSinfo compte 33'000 abonnés ${ }^{6}$. Le hashtag \#19h30 est le mot-dièse dédié aux messages diffusés en lien avec le téléjournal du soir «Le 19h30 ». Le compte @RTSinfo sert à diffuser des messages en lien avec l'actualité. Sur ces deux espaces numériques, la RTS incite les internautes à réagir à l'actualité et à interagir avec les journalistes.

Un processus en trois étapes a été mis en place dans le but de collecter et d'analyser les contenus des messages :

- Étape 1 : Récolte de données en ligne. Le corpus a été construit grâce au moteur de recherche en ligne Topsy. La période d'analyse s'étend du lundi 18 au dimanche 24 novembre 2013. Les éléments de la recherche sont tous les messages contenant le hashtag \#19h30 (240 tweets) ainsi que tous les messages liés au compte@RTSinfo, qu'ils soient émis et retweetés par les gestionnaires du compte@RTSinfo ou qu'ils contiennent la mention @RTSinfo (455 tweets). Un total de 695 tweets a ainsi été récolté.

- Étape 2: Création d'une grille d'observation. Trois unités d'analyse ont été retenues : les acteurs, les contenus des messages et les formes des participations. En premier lieu, il s'agissait

\footnotetext{
${ }^{1}$ Brochure d'entreprise RTS 2014

http://www.rtsentreprise.ch/wp-content/uploads/plaquette-entreprise-RTS.pdf [consulté le 17 septembre 2015]

${ }^{2}$ Dossier de presse audiences 2014

http://www.rtsentreprise.ch/communique_de_presse/bonnes-audiences-2014-pour-la-rts-developpement-spectaculairedu-mobile-et-du-rattrapage-des-videos/ [consulté le 17 septembre 2015].

${ }^{3}$ Site Internet SRG SSR : http://www.srgssr.ch/fr/srg-ssr/entreprise/ [consulté le 17 septembre 2015]

${ }^{4}$ Dossier de presse audiences 2014

http://www.rtsentreprise.ch/communique de presse/bonnes-audiences-2014-pour-la-rts-developpement-spectaculairedu-mobile-et-du-rattrapage-des-videos/ [consulté le 17 septembre 2015]

${ }^{5}$ Ibid.

${ }^{6}$ Ibid.
} 
de caractériser les émetteurs des messages. Dans ce but, le profil associé à chaque tweet a été examiné. Les catégories sont basées sur les indications fournies par les détenteurs des profils eux-mêmes. Ensuite, une typologie des contenus des messages ainsi que des formes de participation (et d'injonction) a été réalisée. Pour chaque unité, une série de catégories exclusives a été créée et des critères de classement ont été conçus.

- Étape 3: Analyse de contenu des messages. Chaque message a été lu et classé dans une catégorie à l'aide du logiciel informatique d'analyse de contenu MaxQDA.

L'étude présentée ici est conçue comme une première approche de la participation journalistique sur Twitter. L'échantillon de données est limité et forcément non représentatif. L'interprétation de nos observations se fait donc avec une prudence particulière. Cependant, nous examinons quelques pistes qui témoignent de dynamiques innovantes dans les processus de production et de réception de l'information en ligne sur un site de microblogging tel que Twitter.

\section{Résultats}

\subsection{Publics et supports matériels}

Les publics participant à l'émission de tweets contenant le \#19h30 et présents sur le compte @RTSinfo ont été classés dans les catégories suivantes : Citoyens (individus n'ayant aucune activité professionnelle dans le domaine du journalisme) ; RTS (différents comptes Twitter de la RTS : @RTSinfo, @Radiotélésuisse, etc.) ; Journalistes (individus indiquant explicitement cette fonction dans leur profil) ; Autres (acteurs comprenant les politiques, les entreprises, les employés de médias et ceux ne remplissant pas les critères ci-dessus).

Les résultats de l'analyse indiquent une forte présence des citoyens, de la RTS ainsi que des journalistes parmi les créateurs de contenus. Les citoyens participent à la création de plus d'un tiers des messages (34.7\% ou 241 messages) ; la Radio Télévision Suisse (RTS) crée 31.1\% des messages (216) et les journalistes créent 25.2\% (175) des messages. Les autres acteurs sont plus faiblement représentés. Parmi les citoyens, quelques individus se distinguent en intervenant à plusieurs reprises dans les deux espaces analysés. Le citoyen le plus présent intervient 11 fois (1.6\% de tous messages) dans notre corpus. Quant aux journalistes, sur 39 individus différents, 4 dominent nettement: le présentateur principal du journal télévisé de la RTS «Le 19h30 » (40 messages ou $5.8 \%$ ) ; le rédacteur en chef de l'actualité télévisée de la RTS (32 ou 4.6\%); un journaliste de la RTS (14 ou $2.0 \%)$; et autre journaliste présent parfois comme présentateur du journal télévisé «Le $19 \mathrm{~h} 30 »(11$ ou 1.6\%). Relevons ainsi que 3 de ces 4 journalistes sont régulièrement - voire très régulièrement - visibles à la télévision.

Il est par ailleurs intéressant d'évoquer la répartition des acteurs selon les deux espaces de discussion. Comme l'indique la Fig. 1, sur le compte @RTSinfo, la RTS représente 44.4\% du total des acteurs, les citoyens représentent $32.5 \%$ et les journalistes $14.3 \%$; sur le hashtag \#19h30, les journalistes représentent $45.8 \%$ des acteurs, les citoyens $38.8 \%$ et la RTS $5.8 \%$. La répartition des citoyens sur ces deux espaces est relativement équivalente. Il y a, en revanche, une différence avérée pour ce qui concerne le nombre d'acteurs de type RTS ainsi que le nombre de journalistes. 


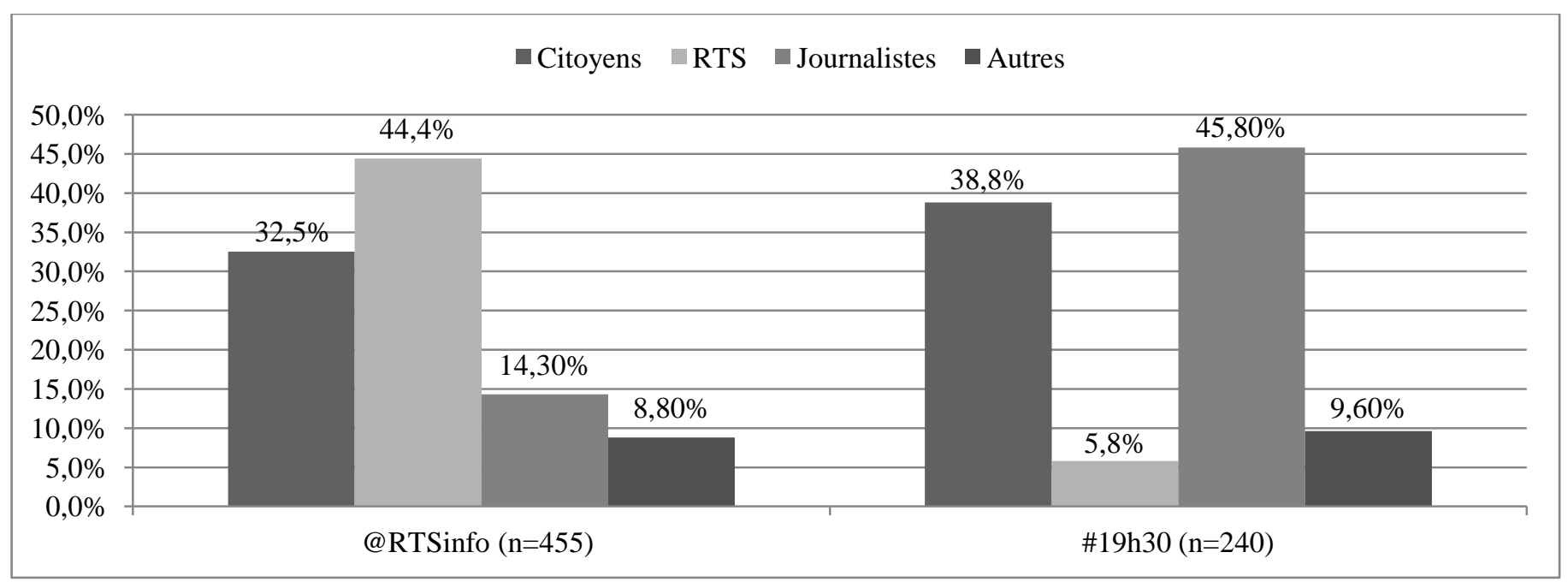

Fig.1. : Pourcentage d'acteurs par fil de discussion ( $n=695)$

Le tableau 1 indique que les citoyens sont majoritairement présents sur le compte @RTSinfo (61.4\%). Cet espace est d'ailleurs celui qui est privilégié par la RTS avec une diffusion de 93.5\% de ses tweets. Seuls les journalistes ont une préférence manifeste pour le hashtag \#19h30 (62.9\%). Il est utile de rappeler ici que la grande présence des journalistes dans les conversations liées au hashtag \#19h30 est assez évidente de par le caractère intrinsèque de cet espace de discussion. Le test du Chicarré indique par ailleurs une relation très significative entre le type d'acteur et le type de fil de discussion $(\mathrm{p}<0.001)$. Par contre, la force de la relation est modérée $(\mathrm{V}=0.31)$.

\begin{tabular}{|c|c|c|c|c|c|c|}
\hline \multirow[t]{2}{*}{ Variable } & \multirow[t]{2}{*}{ Catégorie } & \multicolumn{4}{|c|}{ Type d'acteurs } & \multirow[b]{2}{*}{ Total } \\
\hline & & Citoyens & RTS & Journalistes & Autres & \\
\hline \multirow{2}{*}{$\begin{array}{l}\text { Type de fil de } \\
\text { discussion } * * *\end{array}$} & @RTSinfo & $61.4(148)$ & 93.5 (202) & $37.1(65)$ & $63.5(40)$ & $65.5(455)$ \\
\hline & $\# 19$ h30 & $38.6(93)$ & $6.5(14)$ & $62.9(110)$ & $36.5(23)$ & $34.5(240)$ \\
\hline Total & & $100(241)$ & $100(216)$ & $100(175)$ & $100(63)$ & $100(695)$ \\
\hline \multirow{4}{*}{$\begin{array}{l}\text { Type de } \\
\text { contenu*** }\end{array}$} & Information & $59.3(143)$ & $85.6(185)$ & $58.9(103)$ & $73.0(46)$ & $68.6(477)$ \\
\hline & Injonction & $13.3(32)$ & $12.5(27)$ & $36.0(63)$ & $9.5(6)$ & $18.4(128)$ \\
\hline & Opinion & $24.9(60)$ & $0.5(1)$ & $4.0(7)$ & $11.1(7)$ & $10.8(75)$ \\
\hline & Autre & $2.5(6)$ & $1.4(3)$ & $1.1(2)$ & $6.3(4)$ & $2.2(15)$ \\
\hline Total & & $100.0(241)$ & $100.0(216)$ & $100.0(175)$ & $100.0(63)$ & $100.0(695)$ \\
\hline \multirow[t]{3}{*}{$\begin{array}{l}\text { Type de } \\
\text { participation } * * *\end{array}$} & $\begin{array}{l}\text { Intervention } \\
\text { unique }\end{array}$ & 48.1 (116) & 95.8 (207) & $66.3(116)$ & $58.7(37)$ & $68.5(476)$ \\
\hline & Retweet & $35.3(85)$ & $1.9(4)$ & $29.1(51)$ & $34.9(22)$ & $23.3(162)$ \\
\hline & $\begin{array}{l}\text { Réaction ou } \\
\text { échange }\end{array}$ & $16.6(40)$ & $2.3(5)$ & $4.6(8)$ & $6.3(4)$ & $8.2(57)$ \\
\hline Total & & $100.0(241)$ & $100.0(216)$ & $100.0(175)$ & $100.0(63)$ & $100.0(695)$ \\
\hline
\end{tabular}

Tableau 1 : Relation entre les types d'acteurs et les caractéristiques des messages diffusés (\%). *** p<0.001. Les tests de significativité se rapportent aux mesures d'association Chi-carré et $\mathrm{V}$ de Cramer. La force de la relation type 
d'acteurs/type de fil de discussion est modérée $(\mathrm{V}=0.45)$, tout comme celle de la relation type d'acteurs/type de contenu $(\mathrm{V}=0.25)$ et celle de la relation type d'acteurs/type de participation $(\mathrm{V}=0.31)$.

\subsection{Contenus des messages, types d'injonctions et opinions}

Un des principaux objectifs de notre recherche est également de caractériser le contenu des messages. La classification suivante a été retenue : Information (le message est de nature informative et comprend les partages de nouvelles, d'actualités, d'images et de photos); Injonction (le message est une incitation à faire quelque chose); Opinion (le message est une opinion, une pensée, un jugement...etc.); Autre (le message ne remplit pas les critères ci-dessus).

Comme l'indique le tableau 1, les contenus de type information sont très présents $(68.6 \%$ des messages). Ces tweets représentent d'ailleurs la majorité des messages émis par tous les acteurs de notre échantillon, et notamment pour la RTS (85.6\%). La plus grande partie de ces informations en ligne concernent des faits en lien avec l'actualité. De nature formelle et de ton neutre, leur forme pourrait s'apparenter à de courtes dépêches d'agence de presse. L'étude spécifique des thématiques des messages de type informationnel révèle également, à la fois, une grande variété de sujets originaux traités uniquement une fois, et, en même temps, une concentration de messages autour de grands sujets à portée nationale ou internationale. En effet, la semaine faisant partie de notre échantillon est celle qui correspondait à : la période précédant et comprenant la votation fédérale du 24 novembre 2013 (initiative 1:12 et initiative pour les familles notamment); celle pendant laquelle un otage suisse de Greenpeace a été libéré ; ainsi que celle pendant laquelle des discussions sur le programme nucléaire iranien ont été menées à Genève.

Les injonctions arrivent en deuxième position parmi l'ensemble des messages émis par les différents acteurs (18.4\% des messages totaux). Ces injonctions sont également au second rang des types de contenu les plus diffusés par les journalistes (36.0\%) et par la RTS (12.5\%). Ils représentent, en revanche, seulement le troisième type de contenu utilisé par les citoyens (13.3\%).

Deux types d'injonctions ont été observés dans notre échantillon : les incitations à regarder un média et les incitations à participer. L'injonction à regarder consiste en des messages qui proposent aux individus de consulter une information, une vidéo ou une émission émise par un média. Elle représente la quasi-totalité des messages $(98.5 \%)$. L'injonction à participer prend notamment la forme d'appels à témoins. Celle-ci ne représente que 2 tweets de notre échantillon. Relevons que deux tiers des injonctions ont lieu sur l'espace \#19h30.

Si la catégorie des opinions est relativement peu représentée parmi l'ensemble des messages émis (10.8\% des messages), il est intéressant de noter que les citoyens apprécient ce type de contenu (24.9\% des messages des citoyens). A l'inverse, la RTS et les journalistes utilisent très peu le type de contenu «opinion ».

De façon générale, il existe une relation statistiquement très significative $(\mathrm{p}<0.001)$ entre le type d'acteur et le type de contenu diffusé. La force de cette relation est néanmoins modérée $(\mathrm{V}=0.25)$ (cf. Tableau 1).

\subsection{Formes de participation et d'interaction}

Les formes de participation et d'interaction suivantes ont été distinguées : Intervention unique (message unique qui ne sollicite pas d'interaction); Retweet (message commençant par RT et signifiant un relais d'un message provenant d'un autre utilisateur); Réaction ou échange (message commençant par@ et s'adressant explicitement à un autre utilisateur); Autre (publications ne remplissant pas les critères ci-dessus). 
L'analyse des messages a montré que la grande majorité des participations sont des interventions uniques $(68.5 \%)$. Pour la RTS, ces messages représentent $95.8 \%$ de tous les messages diffusés par le média. Les retweets représentent $23.3 \%$ et les réactions ou échanges ne représentent que $8.2 \%$ des 695 messages analysés. Le Tableau 1 montre que cette distribution est globalement respectée également parmi les acteurs.

La catégorie des messages qui représentent des réactions ou des échanges est celle qui témoigne d'une interaction entre deux ou plusieurs acteurs. Dans notre étude, les acteurs les plus actifs sont les citoyens, avec 40 messages diffusés sur un total de 57. Pour cette catégorie de messages, il est pertinent de distinguer l'émetteur et le destinataire de chaque message afin de mettre en lumière les relations qui peuvent exister entre les différentes catégories d'acteurs.

\begin{tabular}{llll}
\hline Interaction & & \multicolumn{3}{c}{ Occurrences } \\
\hline Citoyen & $\rightarrow$ RTS & 23 & $(40.4 \%)$ \\
Citoyen & $\rightarrow$ Journaliste & 9 & $(15.8 \%)$ \\
Citoyen & $\rightarrow$ Citoyen & 8 & $(14.0 \%)$ \\
RTS & $\rightarrow$ Citoyen & 5 & $(8.8 \%)$ \\
Journaliste & $\rightarrow$ Citoyen & 4 & $(7.0 \%)$ \\
Citoyen & $\rightarrow$ Autre & 2 & $(3.5 \%)$ \\
Journaliste & $\rightarrow$ RTS & 2 & $(3.5 \%)$ \\
Journaliste & $\rightarrow$ Journaliste & 1 & $(1.8 \%)$ \\
Autre & $\rightarrow$ RTS & 1 & $(1.8 \%)$ \\
Autre & $\rightarrow$ Citoyen & 1 & $(1.8 \%)$ \\
Autre & $\rightarrow$ RTS & 1 & $(1.8 \%)$ \\
Total & & 57 & $(100 \%)$ \\
\hline
\end{tabular}

Tableau 2 : Interactions entre les acteurs

Comme indiqué dans le tableau 2, les citoyens sont les acteurs qui initient le plus d'échanges, que ce soit avec la RTS (40.4\%), avec les journalistes (15.8\%) ou avec d'autres citoyens (14.0\%). Toutes les conversations se limitent à une séquence unique composée de deux messages et se rapprochent par conséquent d'une interaction à caractère personnel. Les journalistes et la RTS, ainsi que les autres catégories de notre échantillon semblent peu intéressés par des interactions.

Comme indiqué dans le Tableau 1, la différence entre les quatre groupes d'acteurs distingués est très significative $(\mathrm{p}<0.001)$ : le type d'acteur influence ainsi le type de participation. Cependant, la force de cette relation est modérée $(\mathrm{V}=0.31)$.

\section{Discussion}

\subsection{Citoyens, journalistes et médias : des préférences matérielles quant au support de participation}

Les résultats de l'analyse ont notamment montré que les acteurs de notre recherche ont une certaine préférence pour des interventions sur l'un ou l'autre des espaces de discussion étudiés. Cette préférence peut être expliquée par les caractéristiques propres de ces espaces. En effet, le compte @RTSinfo contient des informations pouvant être considérées comme généralistes. Le profil Twitter indique que ce compte regroupe les «news, breaking news, RT et productions originales ${ }^{7}$. Comme le corroborent nos résultats, nous pouvons donc nous attendre à une grande diversité d'internautes participant à la diffusion de messages sur cet espace. À l'inverse, le hashtag \#19h30 fait plus

${ }^{7}$ Source : Compte Twitter @RTSinfo. https://twitter.com/RTSinfo [consulté le 05.11.14] 
spécifiquement référence au journal télévisé du soir «le 19h30», et les messages contenant ce motdièse sont essentiellement créés par l'équipe rédactionnelle dudit téléjournal.

\subsection{Une injonction sur des supports médiatiques multiples}

L'observation des usages de Twitter met en évidence une approche multicanale des acteurs médiatiques traditionnels. Ceux-ci peuvent, en effet, profiter du temps de latence entre l'annonce d'une actualité sur internet et sa diffusion à la télévision : en utilisant une injonction à regarder une émission d'information qui sera prochainement diffusée à la télévision, ils suscitent le désir des internautes pour regarder cette émission et ils les incitent à participer dès à présent aux échanges en attendant de pouvoir la regarder à la télévision. Sur le mot-dièse \#19h30, il existe un lien entre, d'une part, la très forte implication des journalistes dans les injonctions à participer sur Twitter et à voir les sujets du journal télévisé et, d'autre part, un fort taux de participation de ces citoyens. La stratégie d'injonction à la participation numérique fonctionne donc assez bien. D'autant que les citoyens se font les relais de ces injonctions en les diffusant à leurs propres contacts via des retweets.

\subsection{Opinions}

Les contenus de type «opinions » sont quasi inexistants. Notamment, de la part de la RTS et des journalistes. Ceci démontre que leurs usages des médias sociaux sont relativement normalisés. En présence de leur public et sur les espaces de leur employeur, les journalistes de notre échantillon ne s'autorisent apparemment pas à des échanges trop personnels, mais assurent plutôt leur présence en suivant une stratégie de diffusion massive d'informations. Les citoyens partagent, eux, leurs avis sur Twitter, conformément au but premier de ce site de micro-publications.

\subsection{Des interventions uniques majoritaires et des échanges peu nombreux}

Le nombre élevé d'interventions uniques peut s'expliquer par le fait que les différents acteurs diffusent majoritairement des informations brutes, correspondant à une actualité variée et abondante. Il existe à la fois un pluralisme dans les thématiques des messages diffusés par les acteurs sur les deux espaces analysés et, également, une concentration sur des sujets communs et fédérateurs. Ces derniers sont par ailleurs fortement relayés via des retweets. Nous pouvons faire un parallèle avec les résultats de l'étude de Marty, Rebillard, Smyrnaios et Touboul (2010) sur la variété de l'information en ligne, qui relève que « la concentration est [...] ainsi très forte sur les sujets les plus mis en avant sur la scène médiatique » (Marty \& al. 2010, p. 115). Les sujets faisant l'objet de la plus grande quantité de messages concernent des thématiques qui, à ce moment-là, étaient également très présents dans l'agenda médiatique télévisuel de la RTS.

Notre étude démontre que, d'une manière générale, les acteurs sont peu intéressés par la création d'échanges : ils n'intègrent que peu les conversations amorcées par d'autres individus. Lorsque des échanges ont lieu, les rapports de communication traditionnellement verticaux entre le média, les journalistes et les citoyens sont bouleversés : des interactions ont lieu entre les citoyens et la RTS et entre les citoyens et les journalistes. La frontière relationnelle entre les acteurs médiatiques et les citoyens semble, de plus, très peu marquée par les codes traditionnels de la communication. Nous avons noté la présence d'émoticônes et de points d'exclamations dans plusieurs interactions émises à la fois par des citoyens et par des journalistes.

Néanmoins, ces échanges sont très rares. D'ailleurs, ce sont majoritairement des citoyens qui les initient. Pourtant, il existe une stratégie de la part de la RTS de mettre en contact les journalistes et les citoyens sur les outils de discussion et socialisation en ligne : sur son site internet, la RTS encourage le public à interagir avec ses journalistes via les médias sociaux, dont elle indique les comptes officiels. Nos résultats démontrent que cette stratégie n'est ni véritablement suivie par les 
acteurs médiatiques, ni porteuse. Enfin, la présence de nombreux échanges inter-citoyens montre que les espaces crées par la RTS sur Twitter sont également un lieu propice à des rapports horizontaux.

\section{Conclusion}

À travers notre travail, nous avons exploré les changements accompagnés par les outils sociaux numériques tels que Twitter dans la production des informations par un grand média Suisse-romand ainsi que dans leurs réceptions par les usagers.

L'analyse des messages publiés sur deux supports matériels de Twitter créés par la RTS a révélé comment des acteurs médiatiques et des acteurs non médiatiques pouvaient cohabiter en ligne pour : (i) solliciter la participation journalistique ; (ii) participer à la création de contenus ; et (iii) participer aux interventions numériques en ligne.

Les internautes sont ainsi considérés par les médias traditionnels et les journalistes comme de véritables acteurs du processus de l'information en ligne qu'il faut à la fois exploiter en tant que force créatrice et en tant que relais d'informations vers un large réseau social et un potentiel public. Comme en témoigne notre échantillon, la stratégie de diffusion massive de messages informationnels et uniques ainsi que les incitations à agir - qui consistent en réalité à promouvoir le contenu d'émissions - dévoilent que les acteurs médiatiques se montrent peu intéressés par la création de contenus originaux et d'échanges avec le public. Les citoyens, eux, relaient principalement des informations publiées initialement par le média.

Sur Twitter, la participation des citoyens est, certes, encouragée par les acteurs médiatiques. Ils offrent ainsi la possibilité aux usagers de se socialiser et d'obtenir une forme de reconnaissance à travers leurs productions de contenus (Granjon \& Denouël, 2010). Cependant, cette participation prend rarement la forme de production d'information. En réalité, tout se passe comme si l'incitation à la participation des citoyens sur les espaces de discussion des médias - qui sont d'ailleurs créés par ces médias - servait de prétexte à accroître leur visibilité et à ancrer leur présence sur une multitude d'espaces tiers, nationaux ou internationaux. Sur le site de micro-publications, les parties prenantes sont en effet exposées à l'ensemble de la communauté formée par leurs « abonnés ».

Cette étude est une première exploration du champ des participations journalistiques sur Twitter. Elle augure d'autres travaux qui pourraient prendre en compte une approche qualitative complémentaire à la méthodologie utilisée dans cet article : une consultation des acteurs de la gestion de l'information télévisuelle à l'ère numérique permettrait, par exemple, de confronter leurs pratiques aux résultats de cette enquête.

\section{Références}

Amez-Droz, P. (2015). Médias suisses à l'ère numérique. Lausanne: Presses polytechniques et universitaires romandes.

Badillo, P.-Y. (2015). Usagers et socio-économie des médias. Revue française des sciences de l'information et de la communication, (6).

Badillo, P.-Y., Bourgeois D., \& Asdourian B. (2010). Perspectives des nouveaux champs de la communication des organisations - Éléments à partir du projet européen Fire Paradox. Les cahiers du numérique, 6(4), 167-180.

Blondiaux, L., \& Sintomer, Y. (2002). L’impératif délibératif. Politix, 15(57), 17-35.

Blondiaux, L., \& Fourniau, J.-M. (2011). Un bilan des recherches sur la participation du public en démocratie: beaucoup de bruit pour rien? Participations, 1(1), 8-35. 
Boullier, D. (2004). La fabrique de l'opinion publique dans les conversations télé. Réseaux, 4(126), 57-87.

Bourgeois, D., Clavien, G., Asdourian, B., \& Van Hove, F. (2014). Usages, médias et réseaux sociaux. Perception des contenus des médias suisses-romands. In P.-Y. Badillo. \& D. Roux (Eds.), Le futur est-il «e-media »? (pp. 94-104). Paris : Economica.

Boyd, D., Golder, S., \& Lotan, G. (2010). Tweet, Tweet, Retweet: Conversational Aspects of Retweeting on Twitter. Proceedings of HICSS-42, Persistent Conversation Track. Kauai, HI: IEEE Computer Society.

Bruns, A., \& Burgess, J. (2012). Researching news discussion on Twitter: New methodologies. Journalism Studies, 13(5-6), 801-814.

Cardon, D. (2010). La démocratie Internet: promesses et limites. Paris : Seuil.

Coleman, S. (2012). Making the e-Citizen: A Socio-Technical Approach to Democracy. In S. Coleman \& P. M. Shane (Eds.), Connecting Democracy-Online. Consultation and the Flow of Communication (pp. 379-395). Massachusetts: Massachusetts Institute of Technology.

Coutant, A., \& Stenger, T. (2012). Les médias sociaux : une histoire de participation. Le Temps des médias, 18(1), 76-86.

Dupret, B., Klaus, E., \& Ghazzal, Z. (2010). Commenter l'actualité sur internet. La structure d'intelligibilité d'un forum de discussion arabe. Réseaux, 2(160-161), 285-317.

Flichy, P. (2008). Internet et le débat démocratique. Réseaux, 4(150), 159-185.

Galibert, O. (2010). Engagement éco-citoyen et participation en ligne: entre agir communicationnel et agir stratégique, In B. Cordelier \& G. Gramaccia (Eds.), Organisations, performativité et engagement: Actes du 80e congrès international ACFAS (pp. 138-149). Montréal: Bibliothèque et Archives nationales du Québec.

Galibert, O. (2013). L'injonction participative au débat environnemental en ligne: imaginaires d'Internet, démocratie environnementale et communication engageante. Les enjeux de l'information et de la communication, 1(14/1), 35-49.

Granjon, F., \& Denouël, J. (2010). Exposition de soi et reconnaissance de singularités subjectives sur les sites de réseaux sociaux. Sociologie, 1(1), 25-43.

Hedman, U., \& Djerf-Pierre, M. (2013). The social journalist: Embracing the social media life or creating a new digital divide? Digital Journalism, 1(3), 368-385.

Hedman, U. (2015). J-Tweeters. Digital Journalism, 3(2), 279-297.

Hermida, A. (2010). Twittering the news: The emergence of ambient journalism. Journalism Practice, 4(3), 297-308.

Hermida, A. (2013). \#Journalism. Digital Journalism, 1(3), 295-313.

Java, A., Song, X., Finin, T., \& Tseng, B. (2007). Why we twitter: understanding microblogging usage and communities. Proceedings of the 9th WebKDD and 1st SNA-KDD 2007 workshop on Web mining and social network analysis, 56-65.

Jeanne-Perrier, V. (2010). Parler de la télévision sur Twitter : une « réception » oblique à partir d'une « conversation » médiatique ? Communication \& Langages, (166), 127-147.

Jeanne-Perrier, V. (2012). Agrandir et quitter le nid du local: l'usage de Twitter par les journalistes dans des rédactions de médias régionaux. Sciences de la Société, (84-85), 192-211. 
Jouët, J., \& Rieffel, R. (2015). La sociabilité des journalistes sur Twitter. Entre performativité, ambivalence et multidimensionnalité. Sur le journalisme, 4(1), 18-33.

Kredens, E., \& Rio, F. (2015). La télévision à l'ère numérique: entre pratiques émergentes et reconfiguration de l'objet médiatique. Études de Communication. Langages, Information, Médiations, (44), 15-28.

Kozinets, R. V. (2010). Netnography: doing ethnographic research online. London : Sage.

Lahire, B. (2004). La culture des individus: dissonances culturelles et distinction de soi. Paris : La Découverte.

Lardellier, P., \& Bryon-Portet, C. (2010). Ego 2.0. Les Cahiers Du Numérique, 6(1), 13-34.

Lasorsa, D. L. (2012). Transparency and other journalistic norms on Twitter: The role of gender. Journalism Studies, 13(3), 402-417.

Lasorsa, D. L., Lewis, S. C., \& Holton, A. E. (2012). Normalizing Twitter: Journalism practice in an emerging communication space. Journalism Studies, 13(1), 19-36.

Marres, N. (2012). Material participation: technology, the environment and everyday publics. Palgrave Macmillan.

Marty, E., Rebillard, F., Smyrnaios, N., \& Touboul, A. (2010). Variété et distribution des sujets d'actualité sur Internet. Une analyse quantitative de l'information en ligne. Mots. Les langages du politique, (93), 107-126.

Mercier, A., \& Pignard-Cheynel, N. (2014). Mutations du journalisme à l'ère du numérique: un état des travaux. Revue française des Sciences de l'information et de la communication, (5).

Monnoyer-Smith, L., \& Wojcik, S. (2014). La participation politique en ligne, vers un renouvellement des problématiques? Participations, 1(8), 5-29.

Neuberger, C., Vom Hofe, H. J., \& Nuernbergk, C. (2013). The Use of Twitter by Professional Journalists: Results of a Newsroom Survey in Germany. In K. Weller, A. Bruns, J. Burgess, M. Mahrt, \& C. Puschmann (Eds.), Twitter and Society (pp. 345-357). New York : Peter Lang Publishing Inc.

Noguera-Vivo, J. M. (2013). How open are journalists on Twitter? Trends towards the end-user journalism. Communication \& Society, 26(1), 93-114.

Pélissier, N. \& Diallo, M. D. (2013). Le journalisme est-il soluble dans Twitter ? Enquête sur le choc de deux médiacultures. In N. Pélissier \& G. Gallezot (Eds.), Twitter. Un monde en tout petit ? (pp. 163-178). Paris, L’Harmattan.

Proulx, M., \& Shepatin, S. (2012). Social TV: how marketers can reach and engage audiences by connecting television to the web, social media, and mobile. Hoboken : John Wiley \& Sons.

Rebillard, F., \& Touboul, A. (2010). Promises unfulfilled? "Journalism 2.0", user participation and editorial policy on newspaper websites. Media, Culture \& Society, 32(2), 323-334.

Revers, M. (2014). The Twitterization of News Making: Transparency and Journalistic Professionalism. Journal of Communication, 64(5), 806-826.

Rieder, B., \& Smyrnaios, N. (2012). Pluralisme et infomédiation sociale de l'actualité: le cas de Twitter. Réseaux, (6), 105-139.

Rojot, J. (2005). Théorie des organisations. Paris : Editions ESKA. 
Radio Télévision Suisse. (2014). Dossier de presse audiences 2014. Repéré à http://www.rtsentreprise.ch/communique_de_presse/bonnes-audiences-2014-pour-la-rtsdeveloppement-spectaculaire-du-mobile-et-du-rattrapage-des-videos/

Singer, J. B. (2005). The political j-blogger. 'Normalizing' a new media form to fit old norms and practices. Journalism, 6(2), 173-198.

Sonet, V. (2015). La télévision sur smartphone: ressorts et implications du renouvellement des modalités d'agrégation. Études de Communication. Langages, Information, Médiations, (44), 4762.

Sunstein, C. R. (2001). Republic.com 2.0. Princeton : Princeton University Press.

Touboul, A. (2010). Journalistes et publics, l'annonce d'un mariage de raison. Communication \& Langages, (166), 19-30.

Zeller, F., \& Hermida, A. (2015). When Tradition meets Immediacy and Interaction. The Integration of Social Media in Journalists' Everyday Practices. Sur le journalisme, 4(1), 106-119. 